Eisele, B. \& Wallenfels, K. (1968a). Eur.J. Biochem. 6, 29. Eisele, B. \& Wallenfels, K. (1968b). FE BS Lett. 1, 25.

Kirschner, K., Eigen, M., Bittmann, R. \& Voigt, B. (1966). Proc. natn. Acad. Sci. U.S.A. 56, 1661.

Monod, J., Wyman, J. \& Changeux, J.-P. (1965). J. molec. Biol. 12, 88.

Wallenfels, K. \& Eisele, B. (1968). Eur.J. Biochem. 3, 267. Wallenfels, K. \& Streffer, C. (1966). Biochem. Z. 346, 119.

Kinetic Probes for the Stoicheiometry and Reactivity of Thiol Groups in Enzymes

By H. Gutfreund and C. H. McMurray. (Molecular Enzymology Laboratory, Department of Biochemistry, University of Bristol, Bristol BS8 1TD, U.K.)

The two salient features of enzymes are their ability to recognize specific substrates and the special reactivity of selected groups within the microenvironment of the catalytic site. These properties are likely to be connected, since the catalytic site is not complete until the reactive enzyme-substrate complex has been formed in response to substrate binding. The study of the reactivities of thiol groups of model compounds and enzymes with known three-dimensional structures offers the possibility of correlating reactivity with environment.

It will be illustrated with results from studies on papain, aldolase, haemoglobin, triose phosphate isomerase and glyceraldehyde 3-phosphate dehydrogenase that, in addition to the usual distinction of reactive and non-reactive thiol groups, there is a very wide range of reactivities. The role of different types of thiol groups and the information that can be obtained from precise stoicheiometry will be discussed in terms of enzyme structure and catalysis.

Thiol-blocking reagents that react at different rates with a particular group on a protein will be compared. The advantage of using the very rapidly reacting chromophoric mercurials (McMurray \& Trentham, 1969) with stopped-flow spectrophotometric techniques will be illustrated.

McMurray, C. H. \& Trentham, D. R. (1969). Biochem.J. 115, 913.

\section{The Thiol Groups of Aldolase}

By P. J. Anderson and R. N. Perham. (Department of Biochemistry, University of Cambridge, Cambridge CB2 1QW, U.K.)

The cysteine residues of rabbit muscle aldolase (EC 4.1.2.13) have been reported to be distributed unequally between two types of polypeptide chains (Chan, Morse \& Horecker, 1967). However, recent evidence suggests that the subunits of aldolase are identical with respect to cysteine content (Eagles,
Johnson, Joynson, McMurray \& Gutfreund, 1969; Anderson, Gibbons \& Perham, 1969). The role of the cysteine residues in aldolase is also uncertain (Morse \& Horecker, 1968).

Seven unique carboxymethylcysteine-containing peptides have now been isolated from tryptic digests of rabbit muscle aldolase carboxymethylated with iodo $\left[2{ }^{14} \mathrm{C}\right]$ acetic acid in $8 \mathrm{M}$-urea. These peptides have been characterized by amino acid and endgroup analysis and their location within the polypeptide fragments produced by cyanogen bromide cleavage of the enzyme has been determined. The reaction of native aldolase with $5,5^{\prime}$-dithiobis(2-nitrobenzoic acid), iodoacetamide and $N$-ethylmaleimide showed that a total of three cysteine residues per subunit of mol.wt. 40000 were reactive towards these reagents, and that the modification of these three residues was accompanied by a loss in enzymic activity. Chemical analysis of the modified enzymes demonstrated that the same three thiol groups are involved in the reaction with all these reagents, but that the observed reactivity of a given thiol group varies with the reagent used. Thus one group reacted rapidly and two slowly with $5,5^{\prime}$ dithiobis-(2-nitrobenzoic acid), whereas two groups reacted rapidly and one slowly with iodoacetamide.

One reactive thiol group per subunit could be protected when the modification of the enzyme was carried out in the presence of substrate, fructose 1,6-diphosphate, under which conditions enzymic activity was retained. This thiol group has been identified chemically and, presumably, is at or near the active site. Similarly, limitation of the exposure of the native enzyme to iodoacetamide served to restrict alkylation to two thiol groups and left the enzymic activity unimpaired. The thiol group left unmodified is the same as that protected by substrate during more rigorous alkylation. However, it is now more reactive towards 5,5'-dithiobis-(2nitrobenzoic acid) than in the native enzyme, suggesting some small conformational change has occurred in the modified protein. It is worth noting that preincubation of the enzyme with fructose 1,6-diphosphate, which was subsequently removed by dialysis, caused an irreversible fall in thiol-group reactivity measured in the 5,5'-dithiobis-(2-nitrobenzoic acid) reaction.

We conclude that the aldolase tetramer contains at least 28 cysteine residues. Each subunit appears to be identical with respect to number, location and reactivity of thiol groups.

We are grateful to the Canadian Medical Research Council for the award of a fellowship to P.J.A.

Anderson, P. J., Gibbons, I. \& Perham, R. N. (1969). Eur. J. Biochem. 11, 503.

Chan, W., Morse, D. E \& Horecker, B L. (1967). Proc. natn. Acad. Sci. U.S.A. 57, 1013. 